\title{
User Experience of the U.S. Department of Defense (DoD) Respiratory Disease Dashboard
}

\author{
Jennifer A. Cockrill*1, Alice Y. Tsai ${ }^{1}$, Timothy C. Campbell², Jean-Paul Chretien ${ }^{3}$, Julie A. \\ Pavlin ${ }^{1}$ and Ronald L. Burke ${ }^{1}$
}

'Division of GEIS Operations, Armed Forces Health Surveillance Center, Silver Spring, MD, USA; ${ }^{2}$ Applied Physics Laboratory, Johns Hopkins University, Laurel, MD, USA; ${ }^{3}$ Division of Integrated Biosurveillance, Armed Forces Health Surveillance Center, Silver Spring, MD, USA

\section{Objective}

Evaluate the user experience of a novel electronic disease reporting and analysis system deployed across the DoD global laboratory surveillance network

\section{Introduction}

Lessons learned from the 2009 influenza pandemic have driven many changes in the standards and practices of respiratory disease surveillance worldwide. In response to the needs for timely information sharing of emerging respiratory pathogens (1), the DoD Armed Forces Health Surveillance Center (AFHSC) collaborated with the Johns Hopkins University Applied Physics Laboratory (JHU/APL) to develop an Internet-based data management system known as the Respiratory Disease Dashboard (RDD). The goal of the RDD is to provide the AFHSC global respiratory disease surveillance network a centralized system for the monitoring and tracking of lab-confirmed respiratory pathogens, thereby streamlining the data reporting process and enhancing the timeliness for detection of potential pandemic threats. This system consists of a password-protected internet portal that allows users to directly input respiratory specimen data and visualize data on an interactive, global map. Currently, eight DoD partner laboratories are actively entering respiratory pathogen data into the RDD, encompassing specimens from sentinel sites in eleven countries: Cambodia, Colombia, Kenya, Ecuador, Egypt, Honduras, Nicaragua, Paraguay, Peru, Uganda, and the United States. A user satisfaction survey was conducted to guide further development of the RDD and to support other disease surveillance efforts at the AFHSC.

\section{Methods}

User training was provided to partner laboratories during a transition of data submission from Excel spreadsheet to RDD electronic data entry between November 2011 and May 2012. A user experience survey was distributed to the participating laboratories in August 2012 and based on the experience of 139 entries. The survey adopted elements of the SWOT (Strength-Weaknesses-Opportunities-Threats) analysis to determine the system's strengths and weaknesses as well as to solicit users' perspectives on the efficiency of the system in assisting with disease surveillance data entry and visualization. Questionnaires in an open-ended (free-text response) format were distributed to all eight participating laboratories. Common themes were identified based on the solicited responses.

\section{Results}

Although only four of eight participating laboratory partners replied to the survey (50\% survey response rate), all survey were completed without any omission of questions (100\% completion rate). $2 / 25(8 \%)$ total responses were neutral comments and therefore omitted in the thematic analysis (Table 1). In general, there was a distinct dichotomy in opinion between overseas laboratories and domestic laboratories with regard to the usefulness of the RDD, with overseas laboratories viewing the RDD as more useful than domestic laboratories. A review of the comparison between weekly specimens submitted to the AFHSC via Excel spreadsheet and data entered directly into the RDD revealed misunderstandings about the meaning of the data entry labels in the RDD interface. It was noted by four laboratories that a "Quick Start" user manual would be useful to clarify the definitions of some data labels.

\section{Conclusions}

Overall, this user experience evaluation has identified the needs for additional training on RDD data entry procedures and a "Quick Start" user manual to support the standardization of surveillance definitions. In general, users appreciate the visualization of the global DoD laboratory network data. This evaluation demonstrated the importance of active participation from data contributors and the invaluable organizational support in the development of the RDD as an electronic disease reporting and analysis system.

\section{Keywords}

Outbreak Detection; Disease Surveillance; User Experience Evaluation; Data Management

\section{Acknowledgments}

Sheri Lewis, MPH, JHU/APL Program Manager \& Jose L. Sanchez, MD, $\mathrm{MPH}, \mathrm{AFHSC} /$ Global Emerging Infections Surveillance and Response Systems (GEIS) Operations, STI Pillar Head. This work was supported in part by an appointment to the Postgraduate Research Participation Program at the U.S. Army Public Health Command (USAPHC) administered by the Oak Ridge Institute for Science and Education through an interagency agreement between the U.S. Department of Energy and USAPHC.

\section{References}

1. WHO. Pandemic influenza preparedness framework for the sharing of influenza viruses and access to vaccines and other benefits. Geneva:WHO Press, 2011. http://whqlibdoc.who.int/publications/ 2011/9789241503082_eng.pdf

*Jennifer A. Cockrill

E-mail: Jennifer.Cockrill@us.army.mil 